\title{
Análise das Principais Disfunções Estéticas Corporais Decorrentes da Gravidez
}

\author{
Analysis of the Main Aesthetic Dysfunctions of the Body Resulting from Pregnancy \\ Kleyton Lopes Nobre ${ }^{1}$ \\ Lana Leticia Cardoso de Moraes ${ }^{1}$ \\ Paulo Sérgio Monteiro Nepomuceno ${ }^{1}$ \\ Aymee Lobato Brito ${ }^{1}$ \\ Luciane Lobato Sobral ${ }^{2}$ \\ Márcio Clementino de Souza Santos ${ }^{3}$
}

\section{RESUMO}

Objetivo: Verificar quais são as principais disfunções corporais decorrentes da gravidez e analisar de que modo influenciam a qualidade de vida das mães. Metodologia: Trata-se de uma pesquisa observacional com corte transversal. A coleta de dados teve início após a aprovação do Comitê de Ética em Pesquisa da Universidade da Amazônia. Os dados foram coletados de forma presencial em setembro de 2019, por meio de uma ficha de avaliação e a aplicação do questionário adaptado da CELLUQOL, com questões acerca de algumas disfunções, como celulite, gordura localizada, flacidez tissular e estrias, sendo relacionadas à qualidade de vida das mulheres. Resultados: Foram avaliadas 27 primíparas com idade média de $(31,9 \pm 4,3)$ anos, onde $85,2 \%$ apresentavam gordura localizada e celulite, $66,7 \%$ apresentavam estrias e flacidez tissular. $\mathrm{Na}$ inspeção corporal, os principais locais do corpo com a presença de flacidez e gordura foram o abdômen e os flancos. No tocante à celulite, foram os glúteos e as coxas e das estrias foi o abdômen. Conclusão: A gordura localizada e a celulite são as duas disfunções mais encontradas nas participantes da pesquisa, essas mudanças podem dificultar o convívio social, o ambiente de trabalho e a relação com o seu parceiro, podendo desenvolver um quadro de depressão.

\section{DESCRITORES}

Gravidez. Patologias. Estética. Qualidade de Vida. Fisioterapia. Dermatologia.

\begin{abstract}
Objective: To verify which are the main current body aesthetic dysfunctions of pregnancy and to analyze how it may impact in the quality of life of these mothers. Methodology: It is an observational study with a transversal cut. The data collection began after approval by the Research Ethics Committee of the University of Amazonia. The data were collected in September 2019 through an evaluation form and the adapted CELLUQOL questionnaire containing questions about some dysfunctions, like cellulite, localized fat, flabbiness and stretch marks, being related to quality of life. Results: 27 primiparous women with a mean age of $31.9( \pm 4.3)$ years were evaluated, with $85.2 \%$ presenting localized fat and cellulite, $66.7 \%$ presenting stretch marks, matching the data of tissue sagging. In the body inspection, the main sites of the body with the presence of flabbiness and localized fat were abdomen and flanks. Regarding cellulite, it was the glutes and thighs, and the stretch marks were the abdomen. Conclusion: It was concluded that localized fat and cellulite are the two most common dysfunctions found in the participants and that these changes can make social life, the work environment and the relationship with their partner more difficult and may develop a depression.
\end{abstract}

\section{DESCRIPTORS}

Pregnancy. Pathologies. Aesthetic. Quality of Life. Physiotherapy. Dermatology.

${ }^{1}$ Fisioterapeuta, graduado pela Universidade da Amazônia (UNAMA), Belém, Pará, Brasil.

${ }^{2}$ Docente do Curso de Fisioterapia da Universidade da Amazônia (UNAMA) e da Universidade Estadual do Pará (UEPA), Belém, Pará, Brasil.

${ }^{3}$ Doutor em Doenças Tropicais pela Universidade Federal do Pará - UFPA, Belém, Pará, Brasil. 
A gravidez consiste em um período fisiológico na vida reprodutiva feminina e é caracterizada por mudanças físicas, hormonais e emocionais, nas quais a mulher deve se adaptar. Essas modificações têm como princípio preparar a mulher para gerar um novo ser, proteger o organismo materno, proporcionar o crescimento e a nutrição do feto, além de promover uma melhor recuperação no pós-parto'.

A gravidez afeta praticamente todos os hormônios do corpo e o principal hormônio é a gonadotrofina coriônica humana, mais conhecido como hCG. Ele é exclusivo da gravideze a principal função fisiológica deste hormônio é a de manter o corpo lúteo, de modo que as taxas de progesterona e estrogênio não diminuam, garantindo, assim, a manutenção da gravidez e a ausência de nova ovulação². Além dele, o estrogênio, a progesterona e o lactogênio placentário humano $(\mathrm{Hpl})$, também são responsáveis pelas diversas transformações na gravidez e no período pós-parto ${ }^{3}$.

Essas alterações fisiológicas são responsáveis pelo aparecimento das modificações estéticas no corpo materno e entre as mais comuns, estão: o aparecimento de estrias, a flacidez tissular, o aumento de gordura localizada, o aumento da celulite, dermatoses, hipercromias e a retenção de líquido ${ }^{4}$.

Essas modificações que vão acontecendo no decorrer da gestação tendem a se agravar no pós-parto e, não só afetam o lado estético, mas principalmente, o lado emocional, o que gera bastante preocupação, pois uma baixa na autoestima é um grande passo no caminho de uma futura depressão ${ }^{5}$.

A baixa autoestima se instala ao se olhar no espelho e enxergar um novo corpo diferente do que tinham antes da gravidez, podendo dificultar até mesmo a reintegração no seu ambiente familiar e profissional, por não estarem confortáveis com as modificações que ocorreram ${ }^{6}$. Tendo isso em vista, o objetivo deste trabalho foi verificar quais são as principais disfunções corporais decorrentes da gravidez e analisar de que modo elas influenciam na qualidade de vida dessas mães.

\section{METODOLOGIA}

A pesquisa ocorreu em conformidade com a Resolução nº 196/96, do ConseIho Nacional de Saúde, que regulamenta as pesquisas envolvendo seres humanos, mediante o Parecer de aprovação CAAE 15129019.4.0000.5173, do Comitê de Ética e Pesquisa da Universidade da Amazônia.

Trata-se de um estudo de caráter transversal do tipo observacional, onde participaram da pesquisa 27 mulheres. As informações foram coletadas por meio de um questionário com perguntas fechadas em setembro de 2019.

Os critérios de inclusão para o estudo foram: mulheres com apenas um filho, no período de até um ano após o parto, com idade de até 40 anos, sem histórico familiar de depressão e sem intervenções estéticas no corpo, enquanto o critério de exclusão: mulheres em tratamento estético, com histórico de cirurgia plástica no corpo e/ou fazendo uso de medicamentos antidepressivos.

A coleta de dados foi divulgada por meio das redes sociais e organizada no período da tarde, de forma presencial, onde as participantes foram devidamente esclarecidas quanto aos objetivos do trabalho e sobre os 
riscos e benefícios para a comunidade científica e para a própria participante, ficando livres para participarem ou não. Uma vez aceito, foi assinado o Termo de Consentimento Livre e Esclarecido (TCLE), seguido do preenchimento da ficha da avaliação, na qual que constava perguntas para traçar um perfil sociodemográfico e a inspeção corporal.

Além disso, foi aplicado um questionário adaptado do CELLUQOL (Instrumento de avaliação de qualidade de vida em pacientes com celulite) com sete questões adaptadas do CELLUQOL para celulite, gordura localizada, flacidez tissular e estrias, relacionando a disfunção a qualidade de vida.

Os dados coletados foram apurados e digitados no banco de dados elaborado no software Microssoft Office Excel 2016, posteriormente, foram transformados em gráficos e tabelas para serem examinados. A estatística analítica foi utilizada para avaliar os resultados das variáveis categóricas da amostra, por meio dos testes $\mathrm{G}$ e qui-Quadrado. As estatísticas descritivas foram realizadas utilizando-se o software BioEstat 5.3 .

\section{RESULTADOS}

Neste estudo, um total de 27 mulheres respondeu ao questionário, com média de 31.9 anos de idade. Quanto ao uso de medicamentos e hábitos de vida, não ocorreu uma diferença estatisticamente significante na proporção, como mostra a Tabela 1.

Após a análise acerca da inspeção corporal, concluiu-se que a gordura localizada e a celulite foram as disfunções com maior incidência entre as mulheres, como mostra o Gráfico 1.
Durante a inspeção, foi possível obter dados acerca da localização das disfunções como mostra a Tabela 2.

O resultado dos níveis de incômodo, quando relacionados à qualidade de vida provocados pelas alterações percebidas, está apresentado nos dados da Tabela 3. Em algumas disfunções ocorreram respostas estatisticamente significativas dentro de cada grupo, tendo sido adotado o nível de significância 0,05.

A partir das respostas das participantes, acerca do nível de incomodo em relação a cada uma das disfunções estéticas a elas apresentadas, gerou-se o Gráfico 2.

Pode-se perceber que a maioria se incomoda (em maior ou menor grau) em relação à gordura localizada (82,6\%); em segundo lugar, à flacidez tissular e estrias (valores de $38,9 \%)$; e à celulite $(26,7 \%)$.

A gordura localizada foi a categoria que mais incomodou, por outro lado, mais da metade das participantes $(61,5 \%)$ referiu não se sentir incomodada com a presença de celulites. Participantes com estrias e flacidez tissular, obtiveram porcentagem de $38,9 \%$, ficando abaixo da gordura, em relação ao nível de incômodo.

\section{DISCUSSÃO}

Participaram desta pesquisa 27 primíparas, com média 31,9 \pm 4,3 anos de idade, com proporção significativa de pacientes entre 31 e 35 anos, dado que nos diz que as mulheres estão engravidando cada vez mais tarde e que se mostrou semelhante ao estudo de Aldrighi et al. ${ }^{7}$, que mostra mulheres cada vez mais, optando por uma gravidez tardia, 
Tabela 1. Perfil sociodemográfico das participantes do estudo

\begin{tabular}{l|c|c|c}
\cline { 2 - 4 } Perfil sociodemográfico & $\begin{array}{c}\text { Frequência } \\
\mathrm{n}=27\end{array}$ & $\%$ & p-valor \\
\hline Faixa etária (anos) & & 7,4 & $0.0384^{*}$ \\
\hline$<=25$ & 02 & 29,6 & \\
\hline 26 a 30 & 12 & 44,4 & \\
\hline 31 a $35^{*}$ & 05 & 18,5 & \\
\hline$>35$ & & & 0,4414 \\
\hline Uso de medicamentos & 04 & 14,8 & \\
\hline Corticóide & 02 & 7,4 & \\
\hline Esteróides & 03 & 11,1 & \\
\hline Anti- inflamatórios & 06 & 22,2 & \\
\hline Outros & 16 & 59,3 & \\
\hline Não faz uso & & & 0,8474 \\
\hline Hábitos de vida & 14 & 51,9 & \\
\hline Atividade física & 12 & 44,4 & \\
\hline Bebida alcóolica & 01 & 3,7 & \\
\hline Fumante & & & \\
\hline
\end{tabular}

Fonte: Elaborado pelos autores (2019)

Gráfico 1. Gráfico que mensura a incidência das alterações estéticas corporais

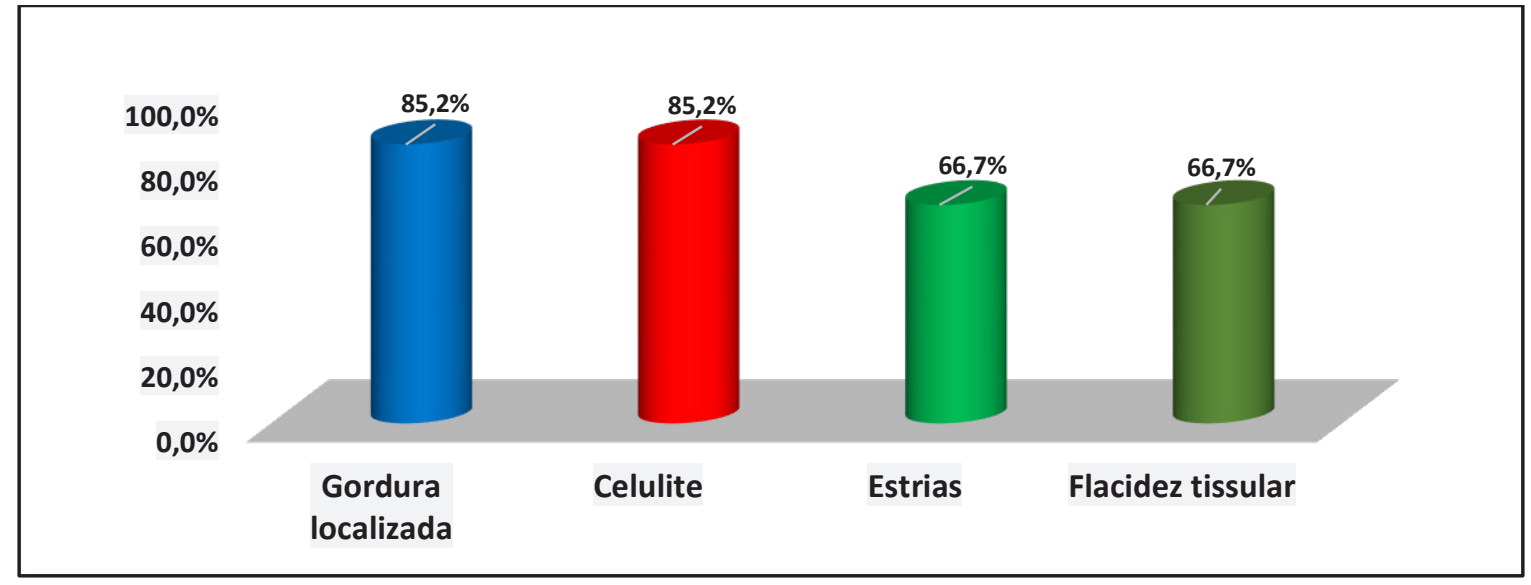

Fonte: Elaborado pelos autores (2019). 
Tabela 2. Tabela de localização das disfunções estéticas

\begin{tabular}{l|c|c|c}
\hline Inspeção Corporal & Frequência & $\%$ & p-valor \\
\hline Localização da flacidez & & & $0.0004^{*}$ \\
Abdômen e flancos & 16 & 88,9 & \\
MMII & 02 & 11,1 & 0,1425 \\
Local das estrias & 07 & 38,9 & \\
Abdômen & 06 & 33,3 & \\
Seios & 04 & 22,2 & \\
MMII & 01 & 5,6 & $<0.0001^{*}$ \\
Costas & 21 & 91,3 & \\
Localização da gordura & 02 & 8,7 & $<0.0001^{*}$ \\
Abdômen e flancos & 19 & 82,6 & \\
MMII & 03 & 13,0 & \\
Localização da celulite & 01 & 4,3 & \\
Glúteos e coxas & & & \\
Abdômen & & & \\
Braços &
\end{tabular}

Fonte: Elaborada pelos autores (2019).

Gráfico 2. Representação do nível de incomodo das participantes

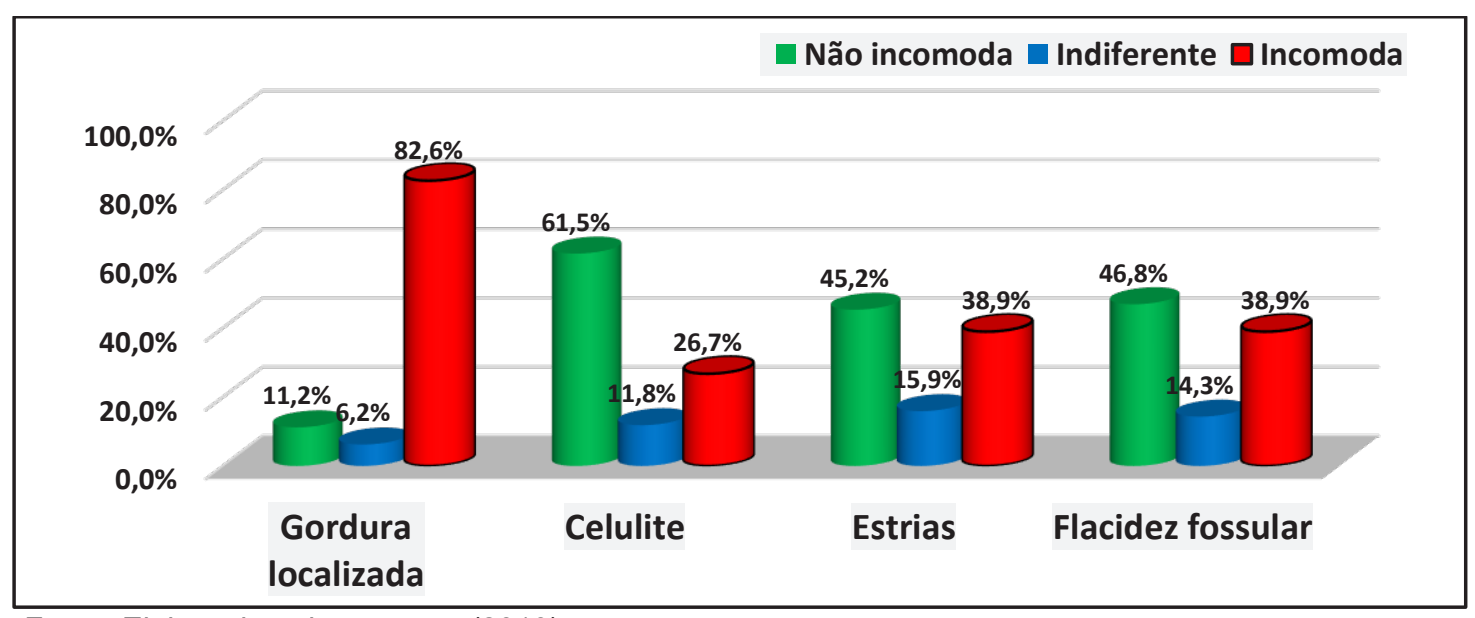

Fonte: Elaborada pelos autores (2019) 
Tabela 3. Tabela de autoavaliação das participantes do estudo

\begin{tabular}{|c|c|c|c|c|c|c|c|c|c|}
\hline \multirow{3}{*}{$\begin{array}{l}\text { Autoavaliação } \\
\text { Aparência do corpo }\end{array}$} & \multirow{2}{*}{\multicolumn{2}{|c|}{$\begin{array}{c}\text { Gordura loca- } \\
\text { lizada }(n=23) \\
n(\%)\end{array}$}} & \multirow{2}{*}{\multicolumn{2}{|c|}{$\begin{array}{c}\begin{array}{c}\text { Celulite } \\
(\mathrm{n}=23)\end{array} \\
\mathrm{n}(\%)\end{array}$}} & \multirow{2}{*}{\multicolumn{2}{|c|}{$\begin{array}{c}\text { Estrias } \\
(\mathrm{n}=18) \\
\mathrm{n}(\%)\end{array}$}} & \multirow{2}{*}{\multicolumn{2}{|c|}{$\begin{array}{c}\text { Flacidez tissu- } \\
\text { lar } \quad(n=18) \\
n(\%)\end{array}$}} & \multirow{3}{*}{$\begin{array}{l}\text { p-valor } \\
0,0126^{*}\end{array}$} \\
\hline & & & & & & & & & \\
\hline & & & & & & & & & \\
\hline Não incomoda & - & - & - & - & - & - & 01 & 5,6 & \\
\hline Não incomoda na maioria das vezes & 02 & 8,7 & 08 & 34,8 & 06 & 33,3 & - & - & \\
\hline Indiferente & - & - & 03 & 13,0 & - & - & 04 & 22,2 & \\
\hline Incomoda na maioria das vezes & 17 & 73,9 & 11 & 47,8 & 09 & 50,0 & 12 & 66,7 & \\
\hline Incomoda o tempo todo & 04 & 17,4 & 01 & 4,3 & 03 & 16,7 & 01 & 5,6 & \\
\hline p-valor & \multicolumn{2}{|c|}{$0,0004^{*}$} & \multicolumn{2}{|c|}{$0,0084^{*}$} & \multicolumn{2}{|c|}{0,2201} & \multicolumn{2}{|c|}{$0,0012^{*}$} & \\
\hline Modo de se vestir & & & & & & & & & $0,0003^{*}$ \\
\hline Não incomoda & - & - & 08 & 34,8 & 02 & 11,1 & 04 & 22,2 & \\
\hline Não incomoda na maioria das vezes & 02 & 8,7 & 06 & 26,1 & 04 & 22,2 & 04 & 22,2 & \\
\hline Indiferente & - & - & 03 & 13,0 & 06 & 33,3 & 02 & 11,1 & \\
\hline Incomoda na maioria das vezes & 17 & 73,9 & 06 & 26,1 & 06 & 33,3 & 08 & 44,4 & \\
\hline Incomoda o tempo todo & 04 & 17,4 & -- & - & - & - & - & - & \\
\hline p-valor & \multicolumn{2}{|c|}{$0,0004^{*}$} & \multicolumn{2}{|c|}{0,5091} & \multicolumn{2}{|c|}{0,4578} & \multicolumn{2}{|c|}{0,2728} & \\
\hline Sua alimentação & & & & & & & & & $<0,0001^{*}$ \\
\hline Não incomoda & 01 & 4,3 & 14 & 60,9 & 06 & 33,3 & 09 & 50,0 & \\
\hline Não incomoda na maioria das vezes & 01 & 4,3 & 04 & 17,4 & 04 & 22,2 & - & - & \\
\hline Indiferente & 01 & 4,3 & 02 & 8,7 & 07 & 38,9 & 06 & 33,3 & \\
\hline Incomoda na maioria das vezes & 17 & 73,9 & 03 & 13,0 & 01 & 5,6 & 03 & 16,7 & \\
\hline Incomoda o tempo todo & 03 & 13,0 & - & - & - & - & - & - & \\
\hline $\mathrm{p}$-valor & \multicolumn{2}{|c|}{$<0,0001^{*}$} & \multicolumn{2}{|c|}{$0,0038^{*}$} & \multicolumn{2}{|c|}{0,1425} & \multicolumn{2}{|c|}{0,2201} & \\
\hline Sua vida sexual & & & & & & & & & $0,0059^{*}$ \\
\hline Não incomoda & 06 & 26,1 & 11 & 47,8 & 02 & 11,1 & 09 & 50,0 & \\
\hline Não incomoda na maioria das vezes & - & - & 08 & 34,8 & 08 & 44,4 & 04 & 22,2 & \\
\hline Indiferente & 01 & 4,3 & 01 & 4,3 & 01 & 5,6 & 01 & 5,6 & \\
\hline Incomoda na maioria das vezes & 15 & 65,2 & 03 & 13,0 & 06 & 33,3 & 04 & 22,2 & \\
\hline Incomoda o tempo todo & 01 & 4,3 & - & - & 01 & 5,6 & - & - & \\
\hline p-valor & \multicolumn{2}{|c|}{$<0,0001^{*}$} & \multicolumn{2}{|c|}{$0,0084^{*}$} & \multicolumn{2}{|c|}{$0,0285^{*}$} & \multicolumn{2}{|c|}{0,0554} & \\
\hline Sentimentos negativos & & & & & & & & & $0,0155^{*}$ \\
\hline Não incomoda & - & - & 03 & 13,0 & 01 & 5,6 & 02 & 11,1 & \\
\hline Não incomoda na maioria das vezes & 03 & 13,0 & 06 & 26,1 & 04 & 22,2 & 05 & 27,8 & \\
\hline Indiferente & - & - & 08 & 34,8 & 02 & 11,1 & 02 & 11,1 & \\
\hline Incomoda na maioria das vezes & 17 & 73,9 & 06 & 26,1 & 10 & 55,6 & 09 & 50,0 & \\
\hline Incomoda o tempo todo & 03 & 13,0 & - & - & 01 & 5,6 & - & - & \\
\hline p-valor & \multicolumn{2}{|c|}{$<0,0001^{*}$} & \multicolumn{2}{|c|}{0,5091} & \multicolumn{2}{|c|}{$0,0109^{*}$} & \multicolumn{2}{|c|}{0,0810} & \\
\hline
\end{tabular}

Fonte: CELLUQOL - Adaptada pelos autores (2019) 
por estarem mais preparadas financeira e psicologicamente. Quanto à prática de hábitos relacionados à qualidade de vida, $51,9 \%$ das participantes praticavam atividades físicas, $44,4 \%$ faziam uso de bebida alcoólica e $96,3 \%$ não eram fumantes, concordando com a pesquisa feita por Malta et al. ${ }^{8}$, onde concluiu-se que ocorreu uma diminuição significativa no consumo de cigarros entre os gêneros masculinos e femininos.

Em relação à percepção das mudanças ocorridas no corpo, o aumento de peso foi a mais frequente, sendo a gordura localizada, a que mais afetou e incomodou as mulheres, resultando em $85,2 \%(n=23)$, dado compatível com a literatura. De acordo com Rebesco et al. ${ }^{9}$, o aumento da camada de gordura é do próprio período gestacional, sobretudo a partir da $10^{a}$ semana, devido as alterações hormonais, causadas pelo estrogênio, provocando uma dificuldade de circulação. Vale ressaltar que a gordura abdominal é importante para a proteção do feto ${ }^{10}$.

Quanto à gordura localizada, a maioria estava localizada no abdômen e flancos, totalizando $91,3 \%(n=21)$ das participantes, dado que coincide com o estudo de Araújo ${ }^{11}$, em que a maioria das mulheres entrevistadas apresentava gordura localizada no abdômen, no momento e após a gravidez.

Paralelo a isso, mais de $70 \%$ das mulheres se incomodavam em relação à alimentação, vida sexual em relação a gordura localizada, confirmando um estudo feito por Bezerra et al. ${ }^{12}$, que mostram que a qualidade de vida das participantes poderia diminuir devido as alterações da gravidez, principalmente, quanto à relação sexual.

Foi notório o alto o número de partici- pantes que relataram ter sentimentos negativos em relação à gordura localizada. Este dado chegou aos escores de 73,9\% (n=17) das participantes, existindo diferença significativa nas respostas $(p<0.0001)$. Tais percentuais, sugerem uma discrepância entre a autoimagem e os padrões veiculados e produzidos culturalmente como aceitos. Esse aspecto estético que repercute, particularmente, em mudanças no vestuário, lazer, sexualidade, dentre outros, como demonstrados nos resultados, corroboram com a pesquisa de Souza et al. ${ }^{13}$, que em um estudo sobre tratamentos estéticos na gravidez, discutiram sobre a procura maciça destes por gestantes, concluindo que as alterações estéticas, tissulares e cutâneas podem constituir preocupação na vida das mulheres, ocasionando sofrimento emocional e diminuição da qualidade de vida.

A celulite também ficou em primeiro lugar como a alteração estética mais encontrada, sendo percebida por $85,2 \%(n=23)$ das participantes, concordando com Afonso et al. ${ }^{14}$, que disseram que entre $85 \%$ e $98 \%$ das mulheres tem ou terão algum grau de celulite, principalmente no período da gestação, com o aumento dos hormônios, em especial, do estrógeno. Em relação à localização, 82,6\% $(n=19)$, apresentavam na região dos glúteos e coxas, concordando com os estudos realizados por Mateus ${ }^{15}$ e Beluco ${ }^{16}$, nos quais as mulheres pesquisadas apresentavam $79,17 \%$ e 67,6 \% de celulite nas áreas dos glúteos e na parte posterior de coxa, respectivamente. Quanto à dor a palpação, apenas 17,4\% $(n=4)$ disseram que sentiam, existindo diferença significativa nas respostas $(p=0.0025)$.

Apesar do grande número de mulheres com essa disfunção, essa não foi a alteração 
mais reclamada, incomodando de alguma forma, apenas $26,7 \%$.

No que se refere à aparência do corpo, alimentação, atividade física com exposição restrita do corpo e vida sexual em relação a celulite, ocorreram diferenças significativas nas respostas, no entanto, em sua maioria, as participantes revelaram ou não se incomodar ou serem indiferentes com a alteração no corpo, indo de encontro a diversos artigos, como o de Cavalcanti et al. ${ }^{17}$, que concluíram que a maioria das participantes apresentava uma autoestima baixa devido a presença da celulite, acarretando inclusive mudança de comportamento. Em outro estudo, Hexsel et al. ${ }^{18}$ relataram que a presença de celulite na vida das mulheres questionadas, em sua maioria, ocasionava atitudes de isolamento e de baixa autoestima, bem como, atuando negativamente sobre a qualidade de vida.

Apesar dos estudos citados acima relatarem uma grande significância em relação aos sentimentos negativos para celulite, no presente estudo, não existiu diferença significativa nas respostas, oscilando desde não incomoda, até incomoda na maioria das vezes, fato esse, que pode estar mudando por conta da desmistificação dos padrões de beleza, principalmente, em publicidades.

Durante a inspeção, as estrias também foram abordadas, onde $66,7 \%(n=18)$ das entrevistadas apresentavam em alguma parte do corpo. Dado esse, relativamente baixo, comparado ao estudo de Souza ${ }^{13}$, que relata que as estrias cutâneas de distensão surgem durante a gestação e são denominadas estrias gravídicas, afetando cerca de $90 \%$ das gestantes. Quanto a sua localização, o estudo de Souza ${ }^{13}$ aponta que a atividade adrenocortical e estrogênica estejam relacionadas com a etiologia das estrias de distensão, ocorrendo associação significativa entre a formação de estrias e o excesso de peso dos bebes e das mães e, por conta disso, a maior parte das estrias estão localizadas no abdômen, concordando com o presente estudo, onde a maioria $38,9 \%(n=7)$ das entrevistadas apresentavam estrias no abdômen, seguido por estrias nos seios.

No tocante a vida sexual e os sentimentos negativos em relação às estrias, ocorreu diferença significativa nas respostas $(p=0,0285$ e $p=0,0109$, respectivamente). No que se refere à vida sexual, $44,4 \%(n=8)$ não se incomodavam na maioria das vezes e $33,3 \%(n=6)$ se incomodam na maioria das vezes, dados que discordam do estudo realizado por Kasielka ${ }^{19}$, que concluiu que a maioria das mulheres se sente insegura ao retornarem a vida sexual com o parceiro, por conta das novas marcas que ficaram no corpo.

Para as estrias, quanto ao sentimento negativo, $55,6 \%(n=10)$ se incomodavam na maioria das vezes, seja por culpa, frustração com o novo corpo ou insegurança com ela mesma e com o parceiro. Nos outros pontos do questionário, como a aparência do corpo, a alimentação, o modo de se vestir e em atividades que impliquem na exposição do corpo, não existiu diferença significativa nas respostas, oscilando desde não incomoda, até incomoda na maioria das vezes.

Por fim, foram analisados os casos de flacidez tissular. A inspeção mostrou que $66,7 \%(n=18)$ das participantes apresentavam flacidez e, dessas, 88,9\% $(n=16)$ flacidez no abdômen, existindo uma proporção significante de pacientes com localização de flacidez no 
abdômen ( $p=0,0004)$. Não foram encontrados muitos dados sobre a localização da flacidez no período gestacional e pós-parto, no entanto, todos os artigos encontrados sobre os tratamentos focavam no abdômen.

Para a flacidez tissular, os escores atingidos foram os menores. Nenhuma participante referiu incomodo o tempo todo quanto ao modo de se vestir, alimentação, vida sexual e sentimentos negativos. Esses dados são relativamente coerentes, uma vez que $51,9 \%$ dos participantes do presente estudo praticavam atividades físicas, de acordo com a recomendação da Sociedade Brasileira de Medicina Esportiva, visto que a prática de atividades físicas auxiliam na manutenção da massa muscular, ocasionando um menor dano de flacidez do tecido ${ }^{20}$.

Em relação à aparência do corpo quanto à flacidez tissular, 66,7\% $(n=12)$ se sentiam incomodadas na maioria das vezes, existindo uma diferença estatística significativa nas respostas $(p=0,0012)$. Esses dados corroboram com os de Demartini et al. ${ }^{21}$, que relatam em estudo que a pele tende não retornar imediatamente ao seu estado inicial após ser submetida a uma alteração temporária, como a gravidez. Porém, quando o limite elástico é ultrapassado, não ocorre o retorno às características originais, então, a fase plástica é caracterizada pela deformação permanente do tecido.

Quando relacionada à vida sexual, $50 \%(n=9)$ responderam que não se incomodavam por ter flacidez tissular, não existindo diferença significativa entre as respostas. Em contrapartida, $50 \%(n=9)$ têm sentimentos negativos em relação a essa alteração. Nessa pesquisa, deparou-se com a escassez de artigos científicos realizados com mulheres grávidas ou após o parto.

Sugere-se, contudo, pesquisas complementares longitudinais, de forma a melhor discutir tais resultados, pois as mudanças no período gestacional e pós-parto são constantes e as variáveis quanto à autoimagem e os sentimentos negativos podem se alterar de forma lábil em todo esse período ${ }^{22}$.

\section{CONCLUSÃO}

Pode-se concluir com o presente estudo, que as disfunções mais citadas e observadas foram a gordura localizada, estrias, celulite e flacidez tissular após o parto, no entanto, a gordura localizada e a celulite foram as duas mais encontradas nas participantes da pesquisa.

A disfunção que mais incomodou foi a gordura localizada, podendo impactar diretamente na qualidade de vida dessas mulheres, afetando seu estado emocional, uma vez que essas mudanças físicas podem dificultar o convívio social, o ambiente de trabalho e a relação com o parceiro.

Sugere-se, portanto, mais estudos acerca das disfunções estéticas causadas pela gravidez e o quanto elas podem interferir na qualidade de vida após o parto, devido à dificuldade de se encontrar estudos atuais relacionados ao tema. 


\section{REFERÊNCIAS}

1. Passos AFMS, Silva JMS, Costa ÍC. Ocorrência de alterações dermatológicas e vasculares em gestantes de uma Unidade Básica de Saúde de Campina Grande, PB. Rev Bras Pesq. Saúde. 2013;15(4): 110-116.

2. Constanzo, L. Fisiologia. $6^{\mathrm{a}}$ ed. Rio de Janeiro: Elsevier; 2018.

3. Tortora G, Derrickson B. Princípios de anatomia e fisiologia. $14^{\mathrm{a}}$ ed. Rio de Janeiro: Guanabara; 2016.

4. Maia M, Marcon CR, Rodrigues SB, Aoki, T. Estrias de distensão na gravidez: fatores de risco em primíparas. An Bras Dermatol. 2009; 84(6):599-605.

5- Belda Júnior W, Di Chiacchio N, Criado PR. Tratado de Dermatologia. $2^{\mathrm{a}}$. edição. São Paulo: Ed. Atheneu; 2015.

6- Silva L, Pessoa F, Pessoa D, Cunha V, Cunha C, Fernandes C. Análise Das Mudanças Fisiológicas Durante A Gestação: Desvendando Mitos. Revista Faculdade Montes Belos. 2015;8(1):1-16.

7- Aldrighi JD, Wall ML, Souza SRRK, Cancela FZV . The experiences of pregnant women at an advanced maternal age: an integrative review. Rev. esc. enferm. USP. 2016; 50(3):512-521

8- Malta DC, Oliveira TP, Luz M, Stopa SR, Junior JBS, Reis AAC. Tendências de indicadores de tabagismo nas capitais brasileiras, 2006 a 2013. Ciênc. Saúde Coletiva. 2015 20(3):631-640.

9- Rebesco DB, Souza WC, Lima VA, Grzelczak MT, Frasson AC, Mascarenhas LPG. Ação do exercício físico na gestação: Um estudo de revisão. Arc Health Invest. 2017 5(6): 311-317.

10- Ribas J, Belló C, Ito C, Mine J, Vellosa J. Alterações metabólicas e inflamatórias na gestação. Rev Ciênc Farm Básica Apl. 2015; 36(2):181-188.

11- Araujo NM, Salim NR, Gualda DMR, Silva LCFP. Corpo e sexualidade na gravidez. Rev Esc Enferm. USP. 2012; 46(3):552-558.

12- Bezerra IFD, Sousa VPS, Santos LC, Viana ESR. Comparação da qualidade de vida em gestantes com disfunção sexual. Rev Bras Ginecol Obstet. 2015;37(6):266-271.

13- Souza A., Paula M., Sobrinho H. Gestação e predisposição ao aparecimento de estrias cutâneas. Universitas: Ciências da Saúde. 2016;14(1):41-52.
14- Afonso JPJM, Tucunduva TCM, Pinheiro MVB, Bagatin E. Celulite: artigo de revisão. Surgical \& Cosmetic Dermatology. Campinas. 2010; 2(3): 214-19.

15- Mateus, A. Caracterização do Fibro Edema Gelóide e Respectivos Tratamentos nos Estudantes de Fisioterapia. Lisboa. Dissertação. Fisioterapia. Escola Superior de Tecnologia de Saúde de Lisboa. Instituto Politécnico de Lisboa. 2014

16- Beluco, L. Perfil dos Paciente em Tratamentos Estéticos para Lipodistrofia e Lipodistrofia Ginóide nos Laboratórios de Cosmetologia e Estética da Universidade do Sul de Santa Catarina no Período de 2012 á 2016. Dissertação. Universidade do Sul de Santa Catarina. 2017.

17- Cavalcanti JM. Bem estar: A visão feminina sobre o fibro edema gelóide. Fisioterapia Brasil. 2013; 14(2):93-98.

18- Hexcel D, Siega C, Schilling-Souza J, Stapenhorst A, Rodrigues TC, Brum C.. Avaliação dos aspectos psicológicos, psiquiátricos e comportamentais de pacientes com celulite: estudo-piloto. Surg Cosmet Dermatol. 2012 $4(2): 131-136$

19- Kasielka A, Sobczak M, Antoszewski B. Risk factors of striae gravidarum. Int J Cosmet Sc. 2015; 37(2):236-240.

20- Montenegro LP. Musculação: Abordagens para a Prescrição e Recomendações para Gestantes. Rev Bras Prescrição de Fisiologia do Exercicio. 2014; 8(47):494-498.

21- Demartini E, Deon KC, Simões NDP. Atuação da Fisioterapia Dermatofuncional na Flacidez Cutânea e Muscular Abdominal em Mulheres no Puerpério: Revisão da Literatura. Rev Bras Terap e Saúde. 2015; 6(1):13-19.

22- Meireles JFF, Neves CM., Carvalho PHB, Ferreira MEC Imagem corporal de gestantes: um estudo longitudinal. Jornal Brasileiro de Psiquiatria. Minas Gerais. Universidade Federal de Juiz de Fora. 2016; 65(3): 223-30.

\section{CORRESPONDÊNCIA}

Aymee Lobato Brito

Av. Almte. Barroso, res. Fernando Guilhon, BI. 10B,

Apto. 303. Souza, Belém-PA, 66613-235

Email: britoaymee20@gmail.com 